\title{
Loi fédérale sur les cellules souches: un équilibre adéquat entre la prudence nécessaire et l'intérêt de la recherche biomédicale
}

\author{
J. Martin
}

Correspondance: Dr Jean Martin, PD La Ruelle 6 CH-1026 Echandens
A l'occasion de référendums notamment, le citoyen suisse est amené à se déterminer sur des objets de plus en plus complexes. Certains de ces objets peuvent susciter des malaises: on se demande si telle loi n'est pas le fruit d'un cerveau de technocrate ou de savant fanatique ... C'est ce genre de crainte qu'exploitent des groupes fondamentalistes qui tendent à voir les choses en noir et blanc alors que la vie est, le plus souvent, faite en réalité de tons de gris. Cela étant, il n'est effectivement pas toujours facile de définir la limite entre l'acceptable et l'inacceptable.

Dans la recherche biomédicale, qui nous occupe à propos de la loi fédérale sur les cellules souches embryonnaires soumise au peuple le 28 novembre 2004, il s'agit de préserver des valeurs éthiques et d'observer le principe de précaution, sans pour autant mutiler les potentiels légitimes de la science. Nous devons être prudents, mais attentifs aussi à ne pas scier la branche sur laquelle nous sommes assis. Or, cela est dit souvent, l'enseignement supérieur et la recherche constituent un créneau essentiel pour la Suisse de demain.

Cette loi a été acceptée par les Chambres en décembre 2003. Il est intéressant de noter que, d'abord, il était envisagé d'avoir un texte régissant la recherche sur les embryons dans leur entier aussi bien que sur les cellules souches qui sont elles des éléments tirés d'embryons; elles peuvent se différencier en plusieurs types de cellules sans avoir le potentiel de se développer en un être humain. Dans une optique de précaution - et dans le sens d'une position de la Commission nationale d'éthique (CNE) dont j'ai l'honneur de faire partie - le Parlement n'a pas inclus dans la loi la recherche sur l'embryon entier. La crainte que certains pourraient exprimer, c'est que cela n'ait été fait que pour éviter des oppositions et que, bientôt, on voudra élargir le champ d'application de la loi à des travaux plus «préoccupants». Il n'y a pas à cela de réponse qui satisfera ceux qui pensent que les chercheurs n'ont pas de morale. Pour moi - tout en étant de ceux qui veulent garder un œil critique sur la science - il importe de ne pas bloquer la re- cherche suisse sur la base d'arguments émotionnels. Etant entendu que des garde-fous adéquats, des limites précises, doivent être mis en place.

Par des travaux sur les cellules souches, on est susceptible de faire des percées utiles au traitement de la maladie de Parkinson, de celle d'Alzheimer ou encore du diabète, affections qui touchent tant de nos concitoyens. Une question majeure est évidemment: mais d'où tirera-t-on ces cellules? Rappelons ici que, suite au développement de la fécondation in vitro (FIVETE), quelques centaines d'embryons congelés devront, en application des dispositions légales en vigueur, être détruits parce qu'ils ne peuvent être implantés dans l'utérus de la femme qui souhaitait un enfant. Pour des raisons diverses: parce que le couple a changé d'avis, que la femme est tombée malade et qu'une grossesse n'est plus souhaitable, voire qu'elle est morte. De tels embryons doivent être décongelés et laissés à leur sort. Dans ces conditions, est-il raisonnable d'interdire de les utiliser pour une recherche strictement surveillée et poursuivant des buts estimables? Ceci alors que de nombreux pays industrialisés permettent de tels travaux (la France elle-même, en général notoirement réticente sur ces sujets, vient de les admettre).

La loi suisse, que je recommande d'accepter, prévoit une série de garanties pour éviter des dérives ainsi que des conflits d'intérêts qui pourraient dévoyer les chercheurs du bon chemin: ainsi le couple d'où est issu l'embryon doit bien entendu donner son consentement éclairé à la recherche; il est précisé qu'on peut le lui demander seulement après que, spontanément, le couple ait décidé d'abandonner l'embryon. Cette décision ne peut donner lieu à aucune rémunération. Les projets de recherche feront l'objet d'un examen serré par une commission d'éthique et doivent obtenir une autorisation de l'Office fédéral de la santé publique, auquel des données précises doivent être fournies ensuite sur le déroulement des travaux. De plus, les chercheurs auront l'obligation de mettre à disposition du grand public un résumé de leurs résultats. Aussi, point très important, une condition 
sine qua non est que des connaissances scientifiques d'égale valeur ne puissent être obtenues d'aucune autre manière que par l'emploi de cellules souches.

Une fois encore, il est vrai que le citoyen qui veut remplir son devoir civique au mieux de sa conscience est confronté à des questions difficiles. Mais il serait très regrettable que des réflexes de peur de la nouveauté (vue comme sys- tématiquement dangereuse ...) fasse rejeter des lois dont le but est d'aller raisonnablement de l'avant. Je crains fort que, si notre pays se laissait dominer par de telles craintes, il ne se recroqueville toujours plus, irrationnellement, avec des conséquences funestes. On peut accepter la loi qui nous est soumise: elle permet une recherche utile tout en l'encadrant d'une manière qui évite des dérapages.

Une discussion sur cette votation fut à l'ordre du jour de la Chambre médicale lors de sa séance du 28 octobre 2004. Le résultat de cette discussion n'était pas encore connu au moment du dernier délai de rédaction.

La Rédcaction

\section{Bundesgesetz über die Forschung an embryonalen Stammzellen: ein angemessenes Gleichgewicht zwischen notwendiger Vorsicht und den Interessen der biomedizinischen Forschung}

\section{J. Martin}

Die Schweizer Bürgerinnen und Bürger müssen insbesondere bei Referendumsabstimmungen über immer komplexere Sachvorlagen entscheiden. Einige davon können Unbehagen auslösen: Man fragt sich, ob bestimmte Gesetze den Köpfen von Technokraten oder fanatischen Wissenschaftlern entsprungen sind. Solche Ängste nutzen dann fundamentalistische Gruppierungen aus, die alles nur schwarzweiss sehen, obwohl das Leben meistens aus Grautönen besteht. Es ist in der Tat nicht immer einfach, eine Grenze zwischen dem Annehmbaren und dem Inakzeptablen zu ziehen.

In der biomedizinischen Forschung, die Gegenstand des am 28. November 2004 zur Abstimmung gelangenden Stammzellenforschungsgesetzes ist, geht es um die Bewahrung der ethischen Werte und die Beachtung des Vorsichtsprinzips, ohne dabei das legitime Potential der Wissenschaft zu beschneiden. Wir müssen vorsichtig sein, aber gleichzeitig auch darauf achten, dass wir nicht an dem Ast sägen, auf dem wir sitzen. Oft heisst es aber, die höhere Bildung und die Forschung stellten eine entscheidende Marktlücke für die Schweiz von morgen.

Dieses Gesetz wurde von den eidgenössischen Räten im Dezember 2003 verabschiedet. Interessant ist, dass zuerst erwogen wurde, in einem einzigen Text die Forschung an den ganzen Embryonen sowie den daraus gewonnenen Stammzellen zu regeln, die sich zu den unterschiedlichen Zelltypen differenzieren, sich jedoch nicht zu einem menschlichen Wesen entwickeln können. Im Sinne einer Vorsichtsmassnahme und gemäss der Haltung der Nationalen Ethikkommission (NEK), der anzugehören ich die Ehre habe, beschloss das Parlament, die Forschung an den Embryonen als Ganzes nicht in dieses Gesetz aufzunehmen. Einige sind vielleicht versucht zu sagen, dass dies nur geschah, 
um Widerstand zu vermeiden und dass man den Geltungsbereich des Gesetzes bald auf «beunruhigendere» Tätigkeiten ausweiten wolle. Darauf gibt es keine Antwort, welche diejenigen zufriedenstellen wird, die glauben, dass den Forschern jegliche Moral fehle. Ich gehöre zu denen, die weiterhin ein kritisches Auge auf die Wissenschaft haben wollen, lege aber Wert darauf, dass die Schweizer Forschung nicht aufgrund emotionaler Argumente blockiert wird. Voraussetzung ist selbstverständlich, dass geeignete Vorsichtsmassnahmen getroffen und genaue Grenzen festgelegt werden.

Dank der Stammzellenforschung werden möglicherweise Durchbrüche bei der Behandlung von Krankheiten wie Parkinson, Alzheimer oder Diabetes erzielt, von denen viele unserer Mitbürgerinnen und Mitbürger betroffen sind. Eine wichtige Frage lautet natürlich: Woher sollen diese Zellen kommen? Zur Erinnerung: Im Anschluss an die Entwicklung der In-vitro-Fertilisation (IVF) müssen gemäss den geltenden gesetzlichen Bestimmungen mehrere hundert eingefrorene Embryonen vernichtet werden, weil sie nicht in die Gebärmutter der Frau eingepflanzt werden können, die sich ein Kind gewünscht hat. Dafür gibt es verschiedene Gründe: Das Paar hat sich anders entschieden, die Frau ist krank geworden und eine Schwangerschaft nicht mehr erwünscht, oder sie ist sogar verstorben. Solche Embryonen müssen aufgetaut und ihrem Schicksal überlassen werden. Ist es unter diesen Umständen vernünftig, ihre Verwendung für eine streng überwachte Forschung mit achtbaren Zielen zu verbieten, während gleichzeitig zahlreiche Industrieländer (sogar das bei solchen Themen als zurückhaltend bekannte Frankreich) solche Forschungsarbeiten zulassen?
Das Schweizer Gesetz, das ich zur Annahme empfehle, sieht eine Reihe von Garantien vor, um Auswüchse und Interessenkonflikte zu verhindern, welche die Forscher vom richtigen Weg abbringen könnten: Das Paar, aus dem der Embryo entstanden ist, muss der Verwendung zu Forschungszwecken nach vorgängiger Aufklärung zustimmen. Es kann erst um die Einwilligung gebeten werden, nachdem es aus freien Stücken beschlossen hat, den Embryo aufzugeben. Diese Entscheidung darf zu keinerlei Entgelt führen. Die Forschungsprojekte werden von einer Ethikkommission streng überwacht und benötigen eine Bewilligung des Bundesamts für Gesundheit, dem anschliessend genaue Angaben über den Ablauf der Arbeiten zu liefern sind. Ausserdem sind die Forscher verpflichtet, eine Zusammenfassung der Ergebnisse öffentlich zugänglich zu machen. Wichtig ist auch die zwingende Bedingung, dass gleichwertige wissenschaftliche Erkenntnisse nicht auf anderem Weg als durch die Verwendung von Stammzellen erlangt werden können.

Es stimmt zwar, dass der Stimmbürger, der seine staatsbürgerliche Pflicht nach bestem Wissen und Gewissen erfüllen will, mit schwierigen Fragen konfrontiert ist. Es wäre aber sehr bedauerlich, wenn die Angst vor dem Neuen (das generell als etwas Gefährliches gesehen wird) dazu führen würde, dass Gesetze abgelehnt werden, die das Ziel haben, mit Vernunft voranzukommen. Wenn sich unser Land von solchen Ängsten beherrschen lässt, befürchte ich stark, dass es sich immer mehr irrational und mit düsteren Folgen abschottet. Das uns vorgelegte Gesetz kann man annehmen: Es ermöglicht eine nützliche Forschung und steckt einen Rahmen ab, mit dem Auswüchse verhindert werden können.

Eine Diskussion über diese Abstimmungsvorlage stand auf der Traktandenliste der Ärztekammer für die Sitzung vom 28. Oktober 2004. Zum Zeitpunkt des Redaktionsschlusses war der Ausgang dieser Diskussion noch nicht bekannt.

Die Redaktion 